Article

\title{
An Optimal Estimate for the Anisotropic Logarithmic Potential
}

\author{
Shaoxiong Hou
}

check for updates

Citation: Hou, S. An Optimal Estimate for the Anisotropic Logarithmic Potential. Mathematics 2022, 10, 261. https://doi.org/ $10.3390 /$ math10020261

Academic Editor: Juan Benigno Seoane-Sepúlveda

Received: 28 December 2021

Accepted: 7 January 2022

Published: 15 January 2022

Publisher's Note: MDPI stays neutral with regard to jurisdictional claims in published maps and institutional affiliations.

Copyright: (c) 2022 by the author. Licensee MDPI, Basel, Switzerland. This article is an open access article distributed under the terms and conditions of the Creative Commons Attribution (CC BY) license (https:// creativecommons.org/licenses/by/ $4.0 /)$.
Key Laboratory of Computational Mathematics and Applications of Hebei Province, College of Mathematical Science, Hebei Normal University, Shijiazhuang 050024, China; sxhou@mail.hebtu.edu.cn

\begin{abstract}
This paper introduces the new annulus body to establish the optimal lower bound for the anisotropic logarithmic potential as the complement to the theory of its upper bound estimate which has already been investigated. The connections with convex geometry analysis and some metric properties are also established. For the application, a polynomial dual log-mixed volume difference law is deduced from the optimal estimate.
\end{abstract}

Keywords: anisotropic log-potential; optimal polynomial inequality; annulus body; dual log-mixed volume

\section{Backgrounds}

The Riesz potential $I_{\alpha}(\alpha>0)$ operator is defined by

$$
I_{\alpha} f(x)=\int_{\mathbb{R}^{n}} \frac{f(y)}{|x-y|^{\alpha}} d y,
$$

where $f$ is a measurable function. It has been widely developed in harmonic analysis including function spaces, mathematical physics and partial differential equations (see [1-4]).

For the endpoint case $\alpha=0$, it is trivial to study the limitation

$$
\lim _{\alpha \rightarrow 0}|x-y|^{-\alpha}=1 \quad \text { as } \quad x \neq y \text {. }
$$

Instead, the convolution kernel is usually changed in such a derivative way

$$
\left.\frac{\partial}{\partial \alpha}|x-y|^{-\alpha}\right|_{\alpha=0}=\left.\frac{\log |x-y|^{-1}}{|x-y|^{\alpha}}\right|_{\alpha=0}=\log |x-y|^{-1} \text { as } x \neq y \text {. }
$$

This logarithmic kernel produces a corresponding logarithmic potential operator, which represents a the better complement for the endpoint case of Riesz potential operator by virtue of effective properties and applications. For example, $|x|^{2-n}(n \geq 3)$ is harmonic on $\mathbb{R}^{n} \backslash o$, while for teh lower dimension $n=2, \log |x|$ is studied since it is harmonic on $\mathbb{R}^{n} \backslash o$ (see $\left.[5,6]\right)$.

Recently, both Riesz potential and logarithmic potential have been studied in an anisotropic way, which is closely related with convex geometry analysis and mathematical physics (see [7-11]). Here we first recall some basic concepts and results in convex geometry.

If the intersection of each line through the origin with a set $K \varsubsetneqq \mathbb{R}^{n}$ is a compact line segment, $K$ is called star-shaped with respect to the origin. Let

$$
\rho_{K}(x)=\max \{\lambda \geq 0: \lambda x \in K\} \quad \text { for } \quad x \in \mathbb{R}^{n} \backslash o \text {, }
$$

where $o$ is the origin, be the radial function of the star-shaped set $K$. $K$ is called a star body with respect to the origin, if $\rho_{K}$ is positive and continuous. We assume that $K$ is a star body 
with respect to the origin and $E$ is a bounded measurable set in this paper. Note that the radial function $\rho_{K}$ is positively homogeneous with degree -1 , i.e.,

$$
\rho_{A}(s x)=s^{-1} \rho_{A}(x) \text { for all } s>0 .
$$

Let $V(E)$ and $E^{c}$ denote, respectively, the $n$-dimensional volume of $E$ and the complement of $E$. We assume $V(E) \neq 0$ in this paper, since when $V(E)=0$, some trivial result follows directly. Let $d S(\cdot)$ denote the natural spherical measure on the boundary $\mathbb{S}^{n-1}$ of the unit ball $B_{2}^{n}$ centered at the origin. Then

$$
V(K)=\frac{1}{n} \int_{\mathbb{S}^{n-1}} \rho_{K}^{n}(u) d S(u) .
$$

Let $\|\cdot\|_{K}$ denote by the Minkowski functional of $K$ :

$$
\|x\|_{K}=\inf \{s>0: x \in s K\} \text { for all } x \in \mathbb{R}^{n}
$$

where

$$
s K=\{s y: y \in K\} .
$$

Note that $\rho_{K}^{-1}(x)=\|x\|_{K}$ and $\|\cdot\|_{B_{2}^{n}}=|\cdot|$, where $|\cdot|$ denotes the Euclidean norm. We refer to $[12,13]$ for more information on convex geometry.

Let $y \in \mathbb{R}^{n}, a>1$ and denote by

$$
R_{a}^{K}(y)=\left\{x \in \mathbb{R}^{n}: \frac{1}{a} \leq\|x-y\|_{K} \leq a\right\}
$$

the $K$-annulus body centered at $y$ with outer radius $a$ and inner radius $\frac{1}{a}$. Then, by the definition of the Minkowski functional, it follows that

$$
V\left(R_{a}^{K}(y)\right)=\left(a^{n}-\left(\frac{1}{a}\right)^{n}\right) V(K) .
$$

Several anisotropic Riesz potentials are introduced and their optimal extreme values estimates are systematically studied in [10]. We omit the details here for the brevity of this paper. Let

$$
P_{\log , m}(K, E ; y)=\int_{E}\left(\log \frac{1}{\|x-y\|_{K}}\right)^{m} d x
$$

be the anisotropic $m$-log-potential of measurable set $E$ at $y \in \mathbb{R}^{n}$ with respect to $K$, and

$$
V_{\log , m}(K, E)=\sup _{y \in \mathbb{R}^{n}} P_{\log , m}(K, E ; y)
$$

be the mixed volume of $K$ and $E$. We refer to [11] for these definitions and [14,15] for their relations with engineering and mathematical physics.

Note that $V_{\log , m}(K, E)$ is obviously an extreme value of the anisotropic $m$-log-potential. It is also closely related to convex geometry analysis. In [11], when $m$ is an odd number, the optimal estimate for $V_{\log , m}(K, E)$ is established as follows:

$$
V_{\log , m}(K, E) \leq \begin{cases}\frac{V(E)}{n^{m}} \sum_{i=0}^{m} \frac{m !}{(m-i) !}\left(\log \frac{V(K)}{V(E)}\right)^{m-i} & \text { for } V(E)>0, \\ 0 & \text { for } V(E)=0 .\end{cases}
$$

When $V(E)>0$, the equality in (2) holds if and only if $E$ is a $K$-ball introduced in [11] up to the difference of a measure zero set. 
For the application of the sharp estimate in (2), the dual polynomial log-Minkowski inequality is established in [11]:

$$
\sum_{i=0}^{m} \frac{n^{m-i} m !}{(m-i) !} \int_{\mathbb{S}^{n-1}}\left(\log \frac{\rho_{K}(u)}{\rho_{L}(u)}\right)^{m-i} d V_{L}(u) \leq \sum_{i=0}^{m} \frac{m !}{(m-i) !}\left(\log \frac{V(K)}{V(L)}\right)^{m-i}
$$

where $m$ is an odd number, $K, L$ are two star bodies and $d V_{L}(\cdot)$ is the normalized conevolume measure

$$
d V_{L}(\cdot)=\left(\frac{\rho_{L}^{n}(\cdot)}{n V(L)}\right) d S(\cdot)
$$

The equality in (3) holds if and only if there exists $s>0$ such that $K=s L$.

Note that (3) generalizes the dual log-Minkowski inequality for a mixed volume of two star bodies (see [12,16]) and produces the polynomial dual for the conjectured $\log$-Minkowski inequality (see [17]).

In this paper, we study the other extreme value of the anisotropic $m$-log-potential:

Definition 1. For $m \in \mathbb{N}$, define

$$
W_{\log , m}(K, E)=\inf _{y \in \mathbb{R}^{n}} P_{\log , m}(K, E ; y) .
$$

Note that because $\log \|x-y\|_{K}^{-1}$ may be negative, $W_{\log , m}(K, E)$ is defined for integer $m$.

In Section 2, some fundamental properties of $W_{\log , m}(K, E)$ are established. Then, in Section 3, we are able to introduce the new annulus body to solve the problem of optimal estimate for $W_{\log , m}(K, E)$ in a precise analytic way. For the application, a polynomial dual log-mixed volume difference law is induced from the optimal estimate.

\section{Fundamental Properties}

First we recall a metric property in [11] for the Minkowski functional of a star body with respect to the origin.

Proposition 1. Let $B_{2}^{n}$ be the unit ball and

$$
\left\{\begin{array}{l}
I_{K}=\sup \left\{\tilde{r} \geq 0: \tilde{r} B_{2}^{n} \subseteq K\right\}, \\
O_{K}=\inf \left\{\tilde{r} \geq 0: K \subseteq \tilde{r} B_{2}^{n}\right\}
\end{array} .\right.
$$

Then

$$
O_{K}^{-1}|x| \leq\|x\|_{K} \leq I_{K}^{-1}|x| \text { for all } x \in \mathbb{R}^{n},
$$

and a quasi-triangle inequality holds for $\|\cdot\|_{K}$

$$
\|x+y\|_{K} \leq I_{K}^{-1} O_{K}\left(\|x\|_{K}+\|y\|_{K}\right) \text { for all } \quad x, y \in \mathbb{R}^{n} .
$$

If $m$ is an even number, the supremum of the anisotropic $m$-log-potential $V_{\log , m}(K, E) \equiv$ $+\infty$ (see [11]). For the infimum of the anisotropic $m$-log-potential $W_{\log , m}(K, E)$, it follows

Proposition 2. $W_{\log , m}(K, E) \equiv-\infty$ for $m$ as an odd number.

Proof. Note that $K$ is a star body with respect to the origin and $E$ is a bounded measurable set. Then $\sup _{x \in E}|x|<+\infty$. For all $C>0$, let $C_{1}=\mathrm{e}^{\left(\frac{C}{V(E)}\right)^{\frac{1}{m}}}>1,|y|>$ $\max \left\{2 O_{K} C_{1}, 2 \sup _{x \in E}|x|\right\}$, where $O_{K}$ is defined in (5). Hence, for all $x \in E$,

$$
\|x-y\|_{K} \geq O_{K}^{-1}|x-y| \geq O_{K}^{-1}(|y|-|x|)>O_{K}{ }^{-1} \frac{|y|}{2}>C_{1}>1 .
$$


Since $m$ is odd, it follows that

$$
\begin{aligned}
P_{\log , m}(K, E ; y) & =\int_{E}\left(\log \frac{1}{\|x-y\|_{K}}\right)^{m} d x \\
& <\int_{E}\left(\log C_{1}^{-1}\right)^{m} d x \\
& =\int_{E}\left(\log \mathrm{e}^{\left(\frac{-C}{V(E)}\right)^{\frac{1}{m}}}\right)^{m} d x \\
& =-C
\end{aligned}
$$

which implies

$$
W_{\log , m}(K, E)=-\infty \quad \text { via } \quad W=\inf _{y \in \mathbb{R}^{n}} P_{\log , m}(K, E ; y)
$$

$W_{\log , m}(K, E)$ has the following metric properties for the nontrivial case ( $m$ is an even number).

Proposition 3. Let $m$ be an even number.

(i) Monotonicity: let $E_{1}$ and $E_{2}$ are bounded measurable sets and $E_{1} \subseteq E_{2}$. Then $W_{\log , m}\left(K, E_{1}\right) \leq$ $W_{\log , m}\left(K, E_{2}\right)$.

(ii) Translation-invariance: for all $z \in \mathbb{R}^{n}$, let $z+E=\{z+y: y \in E\}$. Then $W_{\log , m}(K, z+$ $E)=W_{\log , m}(K, E)$.

(iii) Homogeneity: for all $s>0, W_{\log , m}(s K, s E)=s^{n} W_{\log , m}(K, E)$.

Proof. (i) Since $E_{1} \subseteq E_{2}$, then for all $y \in \mathbb{R}^{n}$,

$$
\int_{E_{1}}\left(\log \frac{1}{\|x-y\|_{K}}\right)^{m} d x \leqslant \int_{E_{2}}\left(\log \frac{1}{\|x-y\|_{K}}\right)^{m} d x
$$

Hence,

$$
\begin{aligned}
W_{\log , m}\left(K, E_{1}\right) & =\inf _{y \in \mathbb{R}^{n}} \int_{E_{1}}\left(\log \frac{1}{\|x-y\|_{K}}\right)^{m} d x \\
& \leqslant \inf _{y \in \mathbb{R}^{n}} \int_{E_{2}}\left(\log \frac{1}{\|x-y\|_{K}}\right)^{m} d x=W_{\log , m}\left(K, E_{2}\right) .
\end{aligned}
$$

(ii) For all $z \in \mathbb{R}^{n}$, by changing the variables $x=z+x_{1}$ and $y=z+y_{1}$, it follows

$$
\begin{aligned}
W_{\log , m}(K, z+E) & =\inf _{y \in \mathbb{R}^{n}} \int_{z+E}\left(\log \frac{1}{\|x-y\|_{K}}\right)^{m} d x \\
& =\inf _{y \in \mathbb{R}^{n}} \int_{E}\left(\log \frac{1}{\left\|x_{1}+z-y\right\|_{K}}\right)^{m} d x_{1} \\
& =\inf _{y_{1} \in \mathbb{R}^{n}} \int_{E}\left(\log \frac{1}{\left\|x_{1}-y_{1}\right\|_{K}}\right)^{m} d x_{1} \\
& =W_{\log , m}(K, E) .
\end{aligned}
$$


(iii) For all $\forall s>0$, by changing the variables $x=s \tilde{x}$ and $y=s \tilde{y}$ and the definition of Minkowski functional in (1), it follows that

$$
\begin{aligned}
W_{\log , m}(s K, s E) & =\inf _{y \in \mathbb{R}^{n}} \int_{s E}\left(\log \frac{1}{\|x-y\|_{s K}}\right)^{m} d x \\
& =\inf _{s \tilde{y} \in \mathbb{R}^{n}} \int_{E}\left(\log \frac{1}{\|s \tilde{x}-s \tilde{y}\|_{s K}}\right)^{m} d s \tilde{x} \\
& =\inf _{\tilde{y} \in \mathbb{R}^{n}} \int_{E}\left(\log \frac{1}{\|\tilde{x}-\tilde{y}\|_{K}}\right)^{m} d s \tilde{x} \\
& =s^{n} W_{\log , m}(K, E) .
\end{aligned}
$$

The continuity of the anisotropic $m$-log-potential $P_{\log , m}(K, E ; \cdot)$ has already been proven in [11]. From this, it follows that

Lemma 1. Let $m$ be an even number. The infimum in

$$
W_{\log , m}(K, E)=\inf _{y \in \mathbb{R}^{n}} P_{\log , m}(K, E ; y)
$$

is achieved at some $y \in \mathbb{R}^{n}$.

Proof. We first conclude that

$$
\lim _{|y| \rightarrow+\infty} P_{\log , m}(K, E ; y)=+\infty
$$

Actually, note that $E$ is a bounded measurable set, then $\sup _{x \in E}|x|<+\infty$. For all $M_{1}>0$, let

$$
|y| \geq \max \left\{2 \sup _{x \in E}|x|, 2 O_{K} \mathrm{e}^{\left(\frac{M_{1}}{V(E)}\right)^{\frac{1}{m}}}\right\}
$$

where $O_{K}$ is defined in (5). It follows from $m$ being an even number and (6) that

$$
\begin{aligned}
P_{\log , m}(K, E ; y) & =\int_{E}\left(\log \frac{1}{\|x-y\|_{K}}\right)^{m} d x \\
& =\int_{E}\left(\log \|x-y\|_{K}\right)^{m} d x \\
& \geq \int_{E}\left(\log \left|O_{K}\right|^{-1}|x-y|\right)^{m} d x \\
& \geq \int_{E}\left(\log \left|O_{K}\right|^{-1}(|y|-|x|)\right)^{m} d x \\
& \geq \int_{E}\left(\log \left(2\left|O_{K}\right|\right)^{-1}|y|\right)^{m} d x \\
& \geq \int_{E}\left(\log \mathrm{e}^{\left(\frac{M_{1}}{V(E)}\right)^{\frac{1}{m}}}\right)^{m} d x \\
& \geq M_{1}
\end{aligned}
$$

which implies that (7) holds.

In the following, we will show that $P_{\log , m}(K, E ; \cdot) \not \equiv+\infty$. As a matter of fact, for $z \in \mathbb{R}^{n}$ and $|z| \geq \sup _{x \in E}|x|$, 


$$
\begin{aligned}
P_{\log , m}(K, E ; z) & =\int_{E}\left(\log \frac{1}{\|x-z\|_{K}}\right)^{m} d x \\
& =\int_{E}\left(\log \|x-z\|_{K}\right)^{m} d x \\
& \leq \int_{E}\left(\log I_{K}^{-1}|x-z|\right)^{m} d x \\
& \leq \int_{E}\left(\log I_{K}^{-1}(|z|+|x|)\right)^{m} d x \\
& \leq \int_{E}\left(\log 2 I_{K}^{-1}|z|\right)^{m} d x \\
& =\left(\log 2 I_{K}^{-1}|z|\right)^{m} V(E) \\
& <+\infty,
\end{aligned}
$$

where $I_{K}$ is in (5). Let $M_{2}=\left(\log 2 I_{K}^{-1}|z|\right)^{m} V(E)$. Because of (7), there exists $D_{1} \geq 0$ such that for all $y \in\left\{y \in \mathbb{R}^{n}:|y|>D_{1}\right\}, P_{\log , m}(K, E ; y)>M_{2}$, which implies that

$$
z \in D=\left\{y \in \mathbb{R}^{n}:|y| \leq D_{1}\right\}
$$

Since $P_{\log , m}(K, E ; \cdot)$ is continuous and $D$ is compact, it can attain its minimum at a point $y_{0}$. Then

$$
P_{\log , m}\left(K, E ; y_{0}\right)=\inf _{y \in D} P_{\log , m}(K, E ; y) \leq P_{\log , m}(K, E ; z) \leq M_{2} \leq \inf _{y \in D^{c}} P_{\log , m}(K, E ; y),
$$

which implies

$$
P_{\log , m}\left(K, E ; y_{0}\right)=\inf _{y \in \mathbb{R}^{n}} P_{\log , m}(K, E ; y)
$$

\section{Optimal Estimate and Application}

Now we are ready to establish the optimal estimate for the infimum of the anisotropic $m$-log-potential.

Theorem 1. Let $m$ be an even number. Then

$$
\begin{aligned}
W_{\log , m}(K, E) \geq & \frac{m ! V(K)}{n^{m}} \sum_{i=0}^{m} \frac{1}{(m-i) !}\left[\log \left(\left(\left(\frac{V(E)}{2 V(K)}\right)^{2}+1\right)^{\frac{1}{2}}+\frac{V(E)}{2 V(K)}\right)\right]^{m-i} \\
& \times\left[\left((-1)^{i}-1\right)\left(\left(\frac{V(E)}{2 V(K)}\right)^{2}+1\right)^{\frac{1}{2}}+\left((-1)^{i}+1\right) \frac{V(E)}{2 V(K)}\right]
\end{aligned}
$$

where the equality holds if and only if $E$ is a K-annulus body with outer radius a and inner radius $\frac{1}{a}$ up to a difference of a measure zero set, namely there exists $y \in \mathbb{R}^{n}$ such that

$$
V\left(E \cap\left(R_{a}^{K}(y)\right)^{c}\right)=V\left(R_{a}^{K}(y) \cap E^{c}\right)=0
$$

where $a=\left(\left(\left(\frac{V(E)}{2 V(K)}\right)^{2}+1\right)^{\frac{1}{2}}+\frac{V(E)}{2 V(K)}\right)^{\frac{1}{n}}$. 
Proof. Let $y \in \mathbb{R}^{n}$ be fixed, and note that $a=\left(\left(\left(\frac{V(E)}{2 V(K)}\right)^{2}+1\right)^{\frac{1}{2}}+\frac{V(E)}{2 V(K)}\right)^{\frac{1}{n}}>1$ and

$$
0<\frac{1}{a}=\left(\left(\left(\frac{V(E)}{2 V(K)}\right)^{2}+1\right)^{\frac{1}{2}}-\frac{V(E)}{2 V(K)}\right)^{\frac{1}{n}}<1
$$

which imply

$$
V\left(R_{a}^{K}(y)\right)=\left[a^{n}-\left(\frac{1}{a}\right)^{n}\right] V(K)=V(E) .
$$

Note that

$$
\begin{aligned}
V\left(E \cap\left(R_{a}^{K}(y)\right)^{c}\right) & =V\left(E \backslash R_{a}^{K}(y)\right) \\
& =V(E)-V\left(R_{a}^{K}(y) \cap E\right) \\
& =V\left(R_{a}^{K}(y)\right)-V\left(R_{a}^{K}(y) \cap E\right) \\
& =V\left(R_{a}^{K}(y) \backslash E\right) \\
& =V\left(R_{a}^{K}(y) \cap E^{c}\right),
\end{aligned}
$$

which, together with the following elementary computations

$$
\left\{\begin{array}{l}
\|x-y\|_{K}>a\left(\text { or }<\frac{1}{a}\right) \text { and }(\log a)^{m}<\left(\log \|x-y\|_{K}\right)^{m} \text { for all } x \in E \cap\left(R_{a}^{K}(y)\right)^{c}, \\
\frac{1}{a} \leqslant\|x-y\|_{K} \leq a \text { and } 0 \leqslant\left(\log \|x-y\|_{K}\right)^{m} \leqslant(\log a)^{m} \text { for all } x \in R_{a}^{K}(y) \cap E^{c},
\end{array}\right.
$$

implies

$$
\begin{aligned}
\int_{R_{a}^{K}(y) \cap E^{c}}\left(\log \|x-y\|_{K}\right)^{m} d x & \leqslant(\log a)^{m} V\left(R_{a}^{K}(y) \cap E^{c}\right) \\
& =(\log a)^{m} V\left(E \cap\left(R_{a}^{K}(y)\right)^{c}\right) \\
& \leqslant \int_{E \cap\left(R_{a}^{K}(y)\right)^{c}}\left(\log \|x-y\|_{K}\right)^{m} d x .
\end{aligned}
$$

Note that $m$ is an even number, then

$$
\begin{aligned}
& P_{\log , m}(K, E ; y) \\
& =\int_{E}\left(\log \frac{1}{\|x-y\|_{K}}\right)^{m} d x \\
& =\int_{E}\left(\log \|x-y\|_{K}\right)^{m} d x \\
& =\int_{\left(R_{a}^{K}(y)\right)^{c} \cap E}\left(\log \|x-y\|_{K}\right)^{m} d x+\int_{R_{a}^{K}(y) \cap E}\left(\log \|x-y\|_{K}\right)^{m} d x \\
& \geq \int_{R_{a}^{K}(y) \cap E^{c}}\left(\log \|x-y\|_{K}\right)^{m} d x+\int_{R_{a}^{K}(y) \cap E}\left(\log \|x-y\|_{K}\right)^{m} d x \\
& =\int_{R_{a}^{K}(y)}\left(\log \|x-y\|_{K}\right)^{m} d x . \\
& =m \int_{\left\{x: \frac{1}{a} \leq\|x-y\|_{K} \leq a\right\}} \int_{1}^{\|x-y\|_{K}} s^{-1}(\log s)^{m-1} d s d x \\
& =m \int_{\left\{x: 1 \leq\|x-y\|_{K} \leq a\right\}} \int_{1}^{\|x-y\|_{K}} s^{-1}(\log s)^{m-1} d s d x \\
& -m \int_{\left\{x: \frac{1}{a} \leq\|x-y\|_{K} \leq 1\right\}} \int_{\|x-y\|_{K}}^{1} s^{-1}(\log s)^{m-1} d s d x \\
& :=I_{1}+I_{2} .
\end{aligned}
$$


Mathematics 2022, 10, 261

8 of 13

By Fubini's theorem, it follows

$$
\begin{aligned}
I_{1} & =m \int_{1}^{a} s^{-1}(\log s)^{m-1} \int_{\left\{x: s \leq\|x-y\|_{K} \leq a\right\}} d x d s \\
& =m \int_{1}^{a} s^{-1}(\log s)^{m-1}\left(a^{n}-s^{n}\right) V(K) d s \\
& =m V(K) a^{n} \int_{1}^{a} s^{-1}(\log s)^{m-1} d s-m V(K) \int_{1}^{a} s^{n-1}(\log s)^{m-1} d s,
\end{aligned}
$$

and

$$
\begin{aligned}
I_{2} & =-m \int_{\frac{1}{a}}^{1} s^{-1}(\log s)^{m-1} \int_{\left\{x: \frac{1}{a} \leq\|x-y\|_{K} \leq s\right\}} d x d s \\
& =-m \int_{\frac{1}{a}}^{1} s^{-1}(\log s)^{m-1}\left(s^{n}-\frac{1}{a^{n}}\right) V(K) d s \\
& =-m V(K) \int_{\frac{1}{a}}^{1} s^{n-1}(\log s)^{m-1} d s+\frac{m V(K)}{a^{n}} \int_{\frac{1}{a}}^{1} s^{-1}(\log s)^{m-1} d s
\end{aligned}
$$

Then, by integration by parts, it follows

$$
\begin{aligned}
I_{1} & +I_{2} \\
= & m V(K)\left[\frac{1}{a^{n}} \int_{\frac{1}{a}}^{1} s^{-1}(\log s)^{m-1} d s+a^{n} \int_{1}^{a} s^{-1}(\log s)^{m-1} d s\right. \\
& \left.-\int_{\frac{1}{a}}^{a} s^{n-1}(\log s)^{m-1} d s\right] \\
= & m V(K)\left[\left.\frac{1}{m a^{n}}(\log s)^{m}\right|_{\frac{1}{a}} ^{1}+\left.\frac{a^{n}}{m}(\log s)^{m}\right|_{1} ^{a}\right. \\
& \left.-(m-1) ! s^{n} \sum_{i=1}^{m} \frac{\left.(-1)^{i-1}(\log s)^{m-i}\right|^{a}(m-i) !}{n^{i}}\right] \\
= & m ! V(K) \sum_{i=0}^{m} \frac{1}{n^{i}(m-i) !}(\log a)^{m-i}\left[-\left(\frac{1}{a}\right)^{n}-(-1)^{i-1} a^{n}\right] \\
= & \left.\frac{m ! V(K)}{n^{m}} \sum_{i=0}^{m} \frac{1}{(m-i) !}\left[\log \left(\left(\frac{V(E)}{2 V(K)}\right)^{2}+1\right)^{\frac{1}{2}}+\frac{V(E)}{2 V(K)}\right)\right]^{m-i} \\
& \times\left[\left((-1)^{i}-1\right)\left(\left(\frac{V(E)}{2 V(K)}\right)^{2}+1\right)^{\frac{1}{2}}+\left((-1)^{i}+1\right) \frac{V(E)}{2 V(K)}\right]
\end{aligned}
$$


Hence, by (10) and (11), it follows that

$$
\begin{aligned}
W_{\log , m}(K, E)= & \inf _{y \in \mathbb{R}^{n}} P_{\log , m}(K, E ; y) \\
= & \inf _{y \in \mathbb{R}^{n}} \int_{E}\left(\log \frac{1}{\|x-y\|_{K}}\right)^{m} d x \\
= & \inf _{y \in \mathbb{R}^{n}} \int_{E}\left(\log \|x-y\|_{K}\right)^{m} d x \\
\geq & \inf _{y \in \mathbb{R}^{n}} \int_{R_{a}^{K}(y)}\left(\log \|x-y\|_{K}\right)^{m} d x \\
= & \frac{m ! V(K)}{n^{m}} \sum_{i=0}^{m} \frac{1}{(m-i) !}\left[\log \left(\left(\left(\frac{V(E)}{2 V(K)}\right)^{2}+1\right)^{\frac{1}{2}}+\frac{V(E)}{2 V(K)}\right)\right]^{m-i} \\
& \times\left[\left((-1)^{i}-1\right)\left(\left(\frac{V(E)}{2 V(K)}\right)^{2}+1\right)^{\frac{1}{2}}+\left((-1)^{i}+1\right) \frac{V(E)}{2 V(K)}\right]
\end{aligned}
$$

To prove the equality in (8), if $E$ is almost a $K$-annulus body up to a difference of a measure zero set, which means there exists $z_{1} \in \mathbb{R}^{n}$ and $a$ such that

$$
V\left(E \cap\left(R_{a}^{K}\left(z_{1}\right)\right)^{c}\right)=V\left(R_{a}^{K}\left(z_{1}\right) \cap E^{c}\right)=0,
$$

which, together with (9), implies

$$
\int_{R_{a}^{K}\left(z_{1}\right) \cap E^{c}}\left(\log \left\|x-z_{1}\right\|_{K}\right)^{m} d x=\int_{E \cap\left(R_{a}^{K}\left(z_{1}\right)\right)^{c}}\left(\log \left\|x-z_{1}\right\|_{K}\right)^{m} d x=0,
$$

and hence

$$
\int_{E}\left(\log \frac{1}{\left\|x-z_{1}\right\|_{K}}\right)^{m} d x=\int_{R_{a}^{K}\left(z_{1}\right)}\left(\log \frac{1}{\left\|x-z_{1}\right\|_{K}}\right)^{m} d x
$$

from (10).

By (10)-(12), it follows

$$
\begin{aligned}
P_{\log , m}\left(K, E ; z_{1}\right)= & \int_{R_{a}^{K}\left(z_{1}\right)}\left(\log \frac{1}{\left\|x-z_{1}\right\|_{K}}\right)^{m} d x \\
= & \frac{m ! V(K)}{n^{m}} \sum_{i=0}^{m} \frac{1}{(m-i) !}\left[\log \left(\left(\left(\frac{V(E)}{2 V(K)}\right)^{2}+1\right)^{\frac{1}{2}}+\frac{V(E)}{2 V(K)}\right)\right]^{m-i} \\
& \times\left[\left((-1)^{i}-1\right)\left(\left(\frac{V(E)}{2 V(K)}\right)^{2}+1\right)^{\frac{1}{2}}+\left((-1)^{i}+1\right) \frac{V(E)}{2 V(K)}\right]
\end{aligned}
$$

which means the equality in (8) holds.

On the other hand, by Lemma 1 , there exists $z_{2} \in \mathbb{R}^{n}, W_{\log , m}(K, E)=P_{\log , m}\left(K, E ; z_{2}\right)$. If $E$ is not a $K$-annulus body up to a difference of a measure zero set, it follows

$$
V\left(E \cap R_{a}^{K}\left(z_{2}\right)^{c}\right) \neq 0 \text { and } V\left(R_{a}^{K}\left(z_{2}\right) \cap E^{c}\right) \neq 0 .
$$

Then the following strict inequality holds from (9):

$$
\int_{R_{a}^{K}\left(z_{2}\right) \cap E^{c}}\left(\log \left\|x-z_{2}\right\|_{K}\right)^{m} d x<\int_{E \cap\left(R_{a}^{K}\left(z_{2}\right)\right)^{c}}\left(\log \left\|x-z_{2}\right\|_{K}\right)^{m} d x,
$$


which implies the inequality in (10) is also strict, and hence

$$
\begin{aligned}
& W_{\log , m}(K, E) \\
& =P_{\log , m}\left(K, E ; z_{2}\right) \\
& =\int_{E}\left(\log \frac{1}{\left\|x-z_{2}\right\|_{K}}\right)^{m} d x \\
& =\int_{E}\left(\log \left\|x-z_{2}\right\|_{K}\right)^{m} d x \\
& >\int_{R_{a}^{K}\left(z_{2}\right)}\left(\log \left\|x-z_{2}\right\|_{K}\right)^{m} d x \\
& =\frac{m ! V(K)}{n^{m}} \sum_{i=0}^{m} \frac{1}{(m-i) !}\left[\log \left(\left(\left(\frac{V(E)}{2 V(K)}\right)^{2}+1\right)^{\frac{1}{2}}+\frac{V(E)}{2 V(K)}\right)\right]^{m-i} \\
& \quad \times\left[\left((-1)^{i}-1\right)\left(\left(\frac{V(E)}{2 V(K)}\right)^{2}+1\right)^{\frac{1}{2}}+\left((-1)^{i}+1\right) \frac{V(E)}{2 V(K)}\right],
\end{aligned}
$$

which means, if the equality in (8) holds, $E$ must be almost a $K$-annulus body up to a difference of a measure zero set.

Remark 1. We claim that there is no such upper bound for $W_{\log , m}(K, E)$ by using $V(K)$ and $V(E)$ as in Theorem 1 when $m$ is an even number.

Proof. Actually, let $V(E)$ be fixed. For all $M>0$, let $E=E_{1} \cup E_{2}$, where $V\left(E_{1}\right)=V\left(E_{2}\right)=$ $2^{-1} V(E)$ and

$$
\operatorname{dist}\left\{E_{1}, E_{2}\right\}=\inf \left\{\left|x_{1}-x_{2}\right| \mid x_{1} \in E_{1}, x_{2} \in E_{2}\right\}>2 O_{K} \mathrm{e}^{\left(\frac{2 M}{V(E)}\right)^{\frac{1}{m}}} .
$$

Then, for all $y \in \mathbb{R}^{n}, \operatorname{dist}\left\{\{y\}, E_{1}\right\}>O_{K} \mathrm{e}^{\left(\frac{2 M}{V(E)}\right)^{\frac{1}{m}}}$ or $\operatorname{dist}\left\{\{y\}, E_{2}\right\}>O_{K} \mathrm{e}^{\left(\frac{2 M}{V(E)}\right)^{\frac{1}{m}}}$. Without loss of generality, suppose $\operatorname{dist}\left\{\{y\}, E_{1}\right\}>O_{K} \mathrm{e}^{\left(\frac{2 M}{V(E)}\right)^{\frac{1}{m}}}$, then, by (6), it follows

$$
\begin{aligned}
P_{\log , m}(K, E ; y) & =\int_{E}\left(\log \frac{1}{\|x-y\|_{K}}\right)^{m} d x, \\
& =\int_{E}\left(\log \|x-y\|_{K}\right)^{m} d x \\
& \geq \int_{E}\left(\log O_{K}^{-1}|x-y|\right)^{m} d x \\
& >\int_{E_{1}}\left(\log O_{K}^{-1}|x-y|\right)^{m} d x \\
& >M,
\end{aligned}
$$

which implies

$$
W_{\log , m}(K, E)=\inf _{y \in \mathbb{R}^{n}} P_{\log , m}(K, E ; y) \geq M .
$$

This completes the proof of the remark.

The infimum of the anisotropic $m$-log-potential is closely related with the convex geometry analysis. For this, a polynomial dual log-mixed volume difference law can be deduced from the optimal estimate for $W_{\log , m}(K, E)$ in Theorem 1. 
Theorem 2. Let $m$ be an even number, $L_{1}, L_{2}, K$ be star bodies with respect to the origin, $L_{1} \subseteq L_{2}$, and $d V_{L_{1}}(u), d V_{L_{2}}(u)$ be the normalized cone-volume measures defined in (4), then

$$
\begin{aligned}
& V\left(L_{2}\right) \int_{\mathbb{S}^{n-1}} \sum_{i=0}^{m} \frac{m !}{n^{i}(m-i) !} \log \left(\frac{\rho_{K}(u)}{\rho_{L_{2}}(u)}\right)^{m-i} d V_{L_{2}}(u) \\
& -V\left(L_{1}\right) \int_{\mathbb{S}^{n-1}} \sum_{i=0}^{m} \frac{m !}{n^{i}(m-i) !} \log \left(\frac{\rho_{K}(u)}{\rho_{L_{1}}(u)}\right)^{m-i} d V_{L_{1}}(u) \geq \\
& \frac{m ! V(K)}{n^{m}} \sum_{i=0}^{m} \frac{1}{(m-i) !}\left[\log \left(\left(\left(\frac{V\left(L_{2}\right)-V\left(L_{1}\right)}{2 V(K)}\right)^{2}+1\right)^{\frac{1}{2}}+\frac{V\left(L_{2}\right)-V\left(L_{1}\right)}{2 V(K)}\right)\right]^{m-i} \\
& \quad \times\left[\left((-1)^{i}-1\right)\left(\left(\frac{V\left(L_{2}\right)-V\left(L_{1}\right)}{2 V(K)}\right)^{2}+1\right)^{\frac{1}{2}}+\left((-1)^{i}+1\right) \frac{V\left(L_{2}\right)-V\left(L_{1}\right)}{2 V(K)}\right],
\end{aligned}
$$

where the equality holds if and only if $L_{2} \backslash L_{1}$ is a K-annulus body centered at origin with outer radius $a$ and inner radius $\frac{1}{a}(a>0)$ up to a difference of a measure zero set.

Proof. Note that $\rho_{K}^{-1}(\cdot)=\|\cdot\|_{K}$, then, by changing to the polar coordinates and integration by parts, it follows that

$$
\begin{aligned}
& P_{\log , m}\left(K, L_{2} \backslash L_{1} ; 0\right) \\
& =\int_{L_{2} \backslash L_{1}}\left(\log \frac{1}{\|x\|_{K}}\right)^{m} d x \\
& =\int_{L_{2}}\left(\log \frac{1}{\|x\|_{K}}\right)^{m} d x-\int_{L_{1}}\left(\log \frac{1}{\|x\|_{K}}\right)^{m} d x \\
& =\int_{L_{2}}\left(\log \rho_{K}(x)\right)^{m} d x-\int_{L_{1}}\left(\log \rho_{K}(x)\right)^{m} d x \\
& =\int_{\mathbb{S}^{n-1}} \int_{0}^{\rho_{L_{2}}(u)} s^{n-1}\left(\log \rho_{K}(s u)\right)^{m} d s d u \\
& -\int_{\mathbb{S}^{n-1}} \int_{0}^{\rho_{L_{1}}(u)} s^{n-1}\left(\log \rho_{K}(s u)\right)^{m} d s d u \\
& =n^{-1} \int_{\mathbb{S}^{n-1}} \int_{0}^{\rho_{L_{2}}(u)}\left(\log \left(s^{-1} \rho_{K}(u)\right)\right)^{m} d s^{n} d u \\
& -n^{-1} \int_{\mathbb{S}^{n-1}} \int_{0}^{\rho_{L_{1}}(u)}\left(\log \left(s^{-1} \rho_{K}(u)\right)\right)^{m} d s^{n} d u \\
& =n^{-1} \int_{\mathbb{S}^{n-1}} \rho_{L_{2}}(u)^{n}\left(\log \frac{\rho_{K}(u)}{\rho_{L}(u)}\right)^{m} d u \\
& +n^{-1} m \int_{\mathbb{S}^{n-1}} \int_{0}^{\rho_{L_{2}}(u)} s^{n-1}\left(\log \left(s^{-1} \rho_{K}(u)\right)\right)^{m-1} d s d u \\
& -n^{-1} \int_{\mathbb{S}^{n-1}} \rho_{L_{1}}(u)^{n}\left(\log \frac{\rho_{K}(u)}{\rho_{L}(u)}\right)^{m} d u \\
& -n^{-1} m \int_{\mathbb{S}^{n-1}} \int_{0}^{\rho_{L_{1}}(u)} s^{n-1}\left(\log \left(s^{-1} \rho_{K}(u)\right)\right)^{m-1} d s d u \\
& =V\left(L_{2}\right) \int_{\mathbb{S}^{n-1}} \sum_{i=0}^{m} \frac{m !}{n^{i}(m-i) !} \log \left(\frac{\rho_{K}(u)}{\rho_{L_{2}}(u)}\right)^{m-i} d V_{L_{2}}(u) \\
& -V\left(L_{1}\right) \int_{\mathbb{S}^{n-1}} \sum_{i=0}^{m} \frac{m !}{n^{i}(m-i) !} \log \left(\frac{\rho_{K}(u)}{\rho_{L_{1}}(u)}\right)^{m-i} d V_{L_{1}}(u),
\end{aligned}
$$


where $d V_{L_{1}}$ and $d V_{L_{2}}$ are defined as in (4).

By Theorem 1, it follows that

$$
\begin{aligned}
& P_{\log , m}\left(K, L_{2} \backslash L_{1} ; 0\right) \\
& =\int_{L_{2} \backslash L_{1}}\left(\log \frac{1}{\|x\|_{K}}\right)^{m} d x \\
& \geq \inf _{y \in \mathbb{R}^{n}} \int_{L_{2} \backslash L_{1}}\left(\log \frac{1}{\|x-y\|_{K}}\right)^{m} d x \\
& \geq \frac{m ! V(K)}{n^{m}} \sum_{i=0}^{m} \frac{1}{(m-i) !}\left[\log \left(\left(\left(\frac{V\left(L_{2} \backslash L_{1}\right)}{2 V(K)}\right)^{2}+1\right)^{\frac{1}{2}}+\frac{V\left(L_{2} \backslash L_{1}\right)}{2 V(K)}\right)\right]^{m-i} \\
& \quad \times\left[\left((-1)^{i}-1\right)\left(\left(\frac{V\left(L_{2} \backslash L_{1}\right)}{2 V(K)}\right)^{2}+1\right)^{\frac{1}{2}}+\left((-1)^{i}+1\right) \frac{V\left(L_{2} \backslash L_{1}\right)}{2 V(K)}\right] \\
& =\frac{m ! V(K)}{n^{m}} \sum_{i=0}^{m} \frac{1}{(m-i) !}\left[\log \left(\left(\left(\frac{V\left(L_{2}\right)-V\left(L_{1}\right)}{2 V(K)}\right)^{2}+1\right)^{\frac{1}{2}}+\frac{V\left(L_{2}\right)-V\left(L_{1}\right)}{2 V(K)}\right)\right]^{m-i} \\
& \quad \times\left[\left((-1)^{i}-1\right)\left(\left(\frac{V\left(L_{2}\right)-V\left(L_{1}\right)}{2 V(K)}\right)^{2}+1\right)^{\frac{1}{2}}+\left((-1)^{i}+1\right) \frac{V\left(L_{2}\right)-V\left(L_{1}\right)}{2 V(K)}\right],
\end{aligned}
$$

which, together with (14), implies (13) holds with the equality holds if and only if $L_{2} \backslash L_{1}$ is a $K$-annulus body centered at origin with outer radius $a$ and inner radius $\frac{1}{a}(a>0)$ up to a difference of a measure zero set.

\section{Conclusions}

Theorem 1 and its Remark 1 complete the systematic study of the optimal upper and lower bounds of the extreme value of the anisotropic $m$-log-potential on a bounded measurable set (for the part of its supremum, we refer to [11]). Note that the anisotropic $m$-log-potential extends the classical logarithmic potential two-fold in anisotropic and higher order of $m$ ways. By virtue of the wide development of Riesz potential with its better complement logarithmic potential for the end point case in harmonic analysis including function spaces, mathematical physics and partial differential equations (see [1-6]), these optimal estimates can be further applied to these related topics.

On the other hand, Brunn-Minkowski inequality and Minkowski inequality including their dual versions and generalizations are main topics in convex geometry analysis (see $[12,13,16,17]$ and their references). The dual log-Minkowski inequality deals with the optimal estimate for mixed volume of two star bodies (see [12,16]), which exists as the dual version for the conjectured log-Minkowski inequality (see [17]). The polynomial dual log-mixed volume difference law in Theorem 2 deduced from the optimal estimate in Theorem 1, deals with the optimal estimate for the difference of mixed volumes of two star bodies, which is totally new and contributes to these theories.

Author Contributions: Conceptualization, S.H.; methodology, S.H.; software, S.H.; validation, S.H.; formal analysis, S.H.; investigation, S.H.; resources, S.H.; data curation, S.H.; writing-original draft preparation, S.H.; writing-review and editing, S.H.; visualization, S.H.; supervision, S.H.; project administration, S.H.; funding acquisition, S.H. All authors have read and agreed to the published version of the manuscript.

Funding: This research was funded by National Natural Science Foundation of China (No.12001157 and 11871191) and Natural Science Foundation of Hebei (No.A2021205013).

Institutional Review Board Statement: Not applicable. 
Informed Consent Statement: Not applicable.

Data Availability Statement: Not applicable.

Acknowledgments: We will like to express our deep thanks to the anonymous referees for their valuable comments.

Conflicts of Interest: The author declares no conflict of interest.

\section{References}

1. Stein, E. Singular Integrals and Differentiability Properties of Functions; Princeton University Press: Princeton, NJ, USA, 1970.

2. Sawano, Y.; Sugano, S.; Tanaka, H. Olsen's inequality and its applications to Schrödinger equations. RIMS Kôkyûroku Bessatsu 2011, B26, 51-80.

3. Liu, L.; Wu, S.; Yang, D.; Yuan, W. New characterizations of Morrey spaces and their preduals with applications to fractional Laplace equations. J. Differ. Equ. 2018, 266, 5118-5167. [CrossRef]

4. Rozenblum, G.; Ruzhansky, M.; Suragan, D. Isoperimetric inequalities for Schatten norms of Riesz potentials. J. Funct. Anal. 2016, 271, 224-239. [CrossRef]

5. Bent, F. The logarithmic potential in higher dimensions. Mat. Fys. Medd. Dan. Cid. Selsk. 1960, 33, 1-14.

6. Hou, S.; Xiao, J. Convex bodies via gravitational potentials. Expo. Math. 2017, 35, 478-482. [CrossRef]

7. Ludwig, M. Anisotropic fractional perimeters. J. Differ. Geom. 2014, 96, 77-93. [CrossRef]

8. Ludwig, M. Anisotropic fractional Sobolev norms. Adv. Math. 2014, 252, 150-157. [CrossRef]

9. Xiao, J.; Ye, D. Anisotropic Sobolev capacity with fractional order. Can. J. Math. 2017, 69, 873-889. [CrossRef]

10. Hou, S.; Xiao, J.; Ye, D. A mixed volume from the anisotropic Riesz-potential. Trans. Lond. Math. Soc. 2018, 5, 71-96. [CrossRef]

11. Hou, S.; Xiao, J. A mixed volumetry for the anisotropic logarithmic potential. J. Geom. Anal. 2018, 28, 2028-2049. [CrossRef]

12. Gardner, R.; Hug, D.; Weil, W.; Ye, D. The dual Orlicz-Brunn-Minkowski theory. J. Math. Anal. Appl. 2015, 430, 810-829. [CrossRef]

13. Schneider, R. Convex Bodies: The Brunn-Minkowski Theory, 2nd ed.; Cambridge University Press: Cambridge, UK, 2014.

14. Fairweather, G.; Johnston, R. The method of fundamental solutions for problems in potential theory. In Treatment of Integral Equations by Numerical Methods; Baker, C., Miller, G., Eds.; Academic Press Inc. (London) Ltd.: London, UK, 1982.

15. Jaswon, M.; Symm, G. Integral Equation Methods in Potential Theory and Elastostatics; Academic Press: London, UK, 1977.

16. Wang, W.; Liu, L. The dual log-Brunn-Minkowski inequalities. Taiwan J. Math. 2016, 20, 909-919. [CrossRef]

17. Böröczky, K.; Lutwak, E.; Yang, D.; Zhang, G. The log-Brunn-Minkowski inequality. Adv. Math. 2012, 231, 1974-1997. [CrossRef] 